\title{
Food, farming, and flu
}

\author{
David Payne digital editor, thebmj.com
}

As we in the northern hemisphere embrace Keats's "season of mists and mellow fruitfulness," gather in the harvest, and reach for the central heating controls, there have been renewed calls for pandemic flu trials in hospitals, owing to the disputed effectiveness of neuraminidase inhibitors.

Earlier this month a report by the Wellcome Trust and Academy of Medical Sciences considered possible future treatments and research priorities (doi:10.1136/bmj.h5390). It drew on recent reviews of the evidence for the use of these drugs to treat and prevent flu.

But have we missed the boat? This week Carl Heneghan and Ben Goldacre (doi:10.1136/bmj.h5635) remind us that because oseltamivir and zanamivir are effectively at the end of their patents, trials will have to be publicly funded, forcing clinicians to rely instead on "often woeful" observational data.

What can we learn from the recent Ebola outbreak? There, trials also happened too late but resulted in clear frameworks and protocols for a future emergency. The Wellcome Trust's director, Jeremy Farrar, who sat on the flu report's steering group, said, "The research protocols and infrastructure need to be put in place now-in 'peace time'-so we can start collecting new evidence immediately at the start of a new epidemic or pandemic."

On the subject of evidence, Public Health England's review of sugar reduction strategies has finally been published (doi:10.

$1136 / \mathrm{bmj} . \mathrm{h} 5675$ ). It said that a sugar tax should be considered but that it would be less effective than other measures.

But will campaigners for a sugar tax face a new setback as a result of planned changes to Europe's common agricultural policy? Emilie Aguirre and colleagues argue (doi:10.1136/bmj. h5085) that the policy, launched in 1962 as the continent emerged from postwar food shortages, has not kept pace with our understanding of nutrition and health.

They warn that scrapping price controls and quotas after 2017 will force Europe's sugar industry to boost production so that it can remain profitable.

Flooding the market with high fructose corn syrup will lead to reformulation of cheaper processed foods and contribute to widening health inequalities in the process. To find out more about high fructose corn syrup, see this week's "60 seconds on" article (doi:10.1136/bmj.h5693).

The European Union is also criticised in a Personal View about changes to continuing professional development (doi:10.1136/ bmj.h5229). Janet Grant and Thomas Zilling are concerned that a recent update to the directive on the recognition of professional qualifications is a tickbox exercise with too much focus on mandatory collection of credits. This will not identify poorly performing doctors or protect patients, they claim.

Attendance at the BMJ Live careers fair in London last week does of course qualify as continuing professional development. The two day event featured an "innovation in practice session," where winners of The BMJ Awards 2015 talked about their projects. This week we launch the 2016 awards (doi:10.1136. bmj.h5665). There are 12 categories. The deadline for entries is 22 January, and the ceremony takes place on 5 May in London.

Meanwhile BMJ's South Asia Awards took place on 30 October in Mumbai. We will have details about the event next week.

We wish all entrants the very best of luck.

Cite this as: BMJ 2015;351:h5768

๑ BMJ Publishing Group Ltd 2015 